\title{
Putting Feelings into Words: Cross-Linguistic Markers of the Referential Process
}

\author{
Sean M. Murphy \\ New York Psychoanalytic \\ Society and Institute \\ Bernard Maskit \\ Mathematics Department \\ Stony Brook University \\ Wilma Bucci \\ 247 East 82nd Street \\ Stony Brook, NY 11794, USA \\ Derner Institute \\ New York, NY 10028, USA \\ daap@optoline.net \\ Adelphi University \\ smurphy1@gmail.com
}

1 Introduction

Emotional states are generally accompanied by the retrieval of specific images and events. Simi-

\begin{abstract}
The use of language to convey emotional experience is of significant importance to the process of psychotherapy, the diagnosis of problems with emotion and memory, and more generally to any communication that aims to evoke a feeling in the recipient. Bucci's theory of the referential process (1997) concerns three phases whereby a person activates emotional, or bodily experience (Arousal Phase), conveys the images and events associated with it (Symbolizing Phase), and reconsiders them (Reorganizing Phase). The Symbolizing Phase is the major focus of this study and is operationalized by a measure called referential activity (RA) based on judges' ratings of the concreteness, specificity, clarity and imagery of language style. Computational models of RA have previously been created in several languages, however, due to the complexity of modeling RA, different modeling strategies have been employed for each language and common features that predict RA across languages are not well understood. Working from previous computational models developed in English and Italian, this study specifies a new model of predictors common to both languages that correlates between $\mathrm{r}$ $=.36$ and. 45 with RA. The components identified support the construct validity of the referential process and may facilitate the development of measures in other languages.
\end{abstract}

lar methods are used to activate emotional states in both research and clinical contexts. For example, appraisal researchers may ask a participant to describe an experience of being angry at oneself in as much vivid detail as possible (Ellsworth and Tong, 2006). Prompting retrieval of images and events also underlies imaginal exposure in treatments for Post Traumatic Stress Disorder (PTSD) and other anxiety disorders (Powers et al., 2010).

Bucci (1997) theorized that the process of putting sensory, visceral and emotional experiences into words requires connection of symbols (words) to non-verbal and non-discrete, analogic (or subsymbolic) information through what she terms the referential process.

\subsection{The referential process.}

Arousal Phase: According to the theory, in the Arousal Phase, material that cannot yet be described or thought of in verbal terms is activated. This may include bodily sensations, or plans for motor action. The speaker is often unsure of what to talk about and may shift loosely from association to association. Language may have greater disfluency (e.g. 'um', 'uh'), more false starts, or non-sequiturs. The proportion of disfluency in a text is used as a major indicator of the Arousal Phase.

Symbolizing Phase: As the activated material is processed it coalesces into a prototypic im- 
age or plan for action. This is the preliminary part of the Symbolizing Phase, when the material is brought into symbolic form but is not yet expressed in language. The latter part of this phase is the expression of this material in words by telling about an image, or event. The language of this phase will tend to be more specific, will focus on describing a single idea or frame, will refer more to the concrete or sensate properties of things, and will tend to evoke imagery in the listener, or reader. This style of language has been operationalized by referential activity (RA), which is understood as the connection of words to nonverbal experience, including bodily and emotional experiences that are subsymbolic. This measure is the focus of the current study and will be discussed in greater detail below along with computational models of referential activity.

Reorganizing Phase: Once symbolically expressed, such ideas are open for reflection and reorganization which takes place during the Reorganizing Phase. The restructuring that occurs during this phase encourages psychological development, and can begin the referential process anew by raising new questions, thoughts or feelings in response to the revelations that have occurred. The language of this phase is marked by references to cognitive processes, logical operations and reasoning. Such references are operationalized by a dictionary of 'Reflection' words (REF) which is used as a measure of the reorganizing phase and is discussed in greater detail below and in Maskit et al. (2015, this conference).

\subsection{Referential activity (RA).}

Referential activity was first operationalized by Bucci through a scoring system whereby judges rate language segments on dimensions of concreteness, specificity, clarity and imagery. Each scale is scored from zero to ten. The four dimensions are significantly inter-correlated for most speakers and most texts and are understood as reflecting different aspects of the same general dimension. For this reason, the average of the four scales is taken as the overall referential activity (RA) score of the segment (Bucci et al.,
2004). Using these four scales, raters are able to achieve excellent inter-rater reliability with standardized item alphas exceeding .90 (Bucci et al., 2004, p. 24). The RA scales (as defined above) have been used in many studies (e.g. Appelman, (2000); Bucci, (1984); Dodd \& Bucci, (1987); Fretter, Bucci, Broitman, Silberschatz, \& Curtis, J., (1994); Horowitz, et al. (1993); Jepson \& Bucci (1999); Langs, Bucci, Udoff, Cramer, \& Thomson, (1993); Watson, J., (1996); also see Samstag, (1997) for a review of dissertations utilizing these scales).

\subsection{The referential process and psychotherapy process.}

In order to function well in life we need to be able to connect our sensory and emotional experiences to the people and events of our life so that we can make the best possible judgments regarding how to respond. We need to take in new information and modify our understanding of the world and our relationships as we and our life situations change.

In talk therapies, Bucci argues that psychological change occurs through the referential process. In the Arousal Phase, activation of a problematic experience can be gradually tolerated as the relationship develops between therapist and patient. In the Symbolizing Phase, the person talks about an episode of life, a dream or fantasy that is connected to this problematic experience. The representation of the experience in share-able symbolic form allows for new information to be brought in and connected with it. Once shared, there is an opportunity for a Reorganizing Phase where the meaning of the events may be reconsidered and further explored.

\subsection{Clinical studies of the referential process.}

Clinical studies have demonstrated that measures of the referential process are meaningful predictors of good psychotherapy process and outcome. Bucci and Maskit (2007) showed that the referential activity of a series of sessions in a single case design of a psychoanalytic treatment had strong correlations with the process ratings of expert clinicians who read and scored 
these sessions. Higher RA in therapist process notes, understood as indicating greater emotional engagement of the therapist, was associated with better treatment outcome as assessed by independent raters (Bucci et al., 2012). Mariani et al. (2013) found that RA (measured by the Italian Weighted Referential Activity Dictionary IWRAD) increased over the course of three psychotherapy treatments that showed improvement in personality functioning.

\subsection{RA as an indicator of episodic memory capability and impairment.}

In their 2008 paper, Nelson, Moskowitz and Steiner found a robust correlation $\left(r_{s}(53)=\right.$ $.69, p<.001)$ between a measure of narrativity and the WRAD in a sample of high school students talking about their most stressful time. In their paper, Nelson et al. (2008) noted the similarity between the "story-now grammar" of narratives (Clark, 1996; Fleischman, 1990) and Tulving's concept of episodic memory as "time travel" (1972). Maskit et al. (2014) directly compared WRAD with measures of episodic memory strength using data provided by Schacter and scored by Addis, Wong and Schacter (2008), and also found strong correlations between the two measures. In line with these findings WRAD has been shown to differentiate populations with impairments in episodic memory such as participants with Schizophrenia (Lewis et al., 2009) and with Alzheimer's dementia (Nelson and Polignano, 2009) from nonclinical controls.

\subsection{Previous computational models of referential activity.}

The first computerized model of RA was the Computerized Referential Activity (CRA) of Mergenthaler and Bucci (1999), based on modeling very high and very low RA scale scores. The technique used to develop this measure was refined to develop the second generation computer model, the Weighted Referential Activity Dictionary (WRAD) (Bucci and Maskit, 2006). The WRAD was developed through a process of modeling the frequency with which words appeared in texts at several levels of RA as scored by judges, producing a weighted dictionary. In comparison to the CRA the WRAD represents an improvement in the correlation between computerized and judge based RA scoring in six of the seven samples utilized to model and test the dictionary. Correlations between WRAD and judges scoring of RA ranged between $r=.38$ and $r=.60$ in these samples. A detailed explanation of the modeling procedure for the WRAD dictionary may be found in Bucci and Maskit (2006).

The WRAD is a weighted dictionary with weights lying between -1 , for words used more frequently in low RA speech, and +1 for words used more frequently in high RA speech. The WRAD comprises 697 frequently used items, primarily function words, including personal pronouns such as 'she', conjunctions such as 'and', and articles such as 'the'. Because of the dominance of such extremely frequent function words, the dictionary covers approximately $85 \%$ of the tokens used in the sample from which the dictionary was derived.

The structure of the Italian language is different from that of English; thus modeling strategies based on a restricted number of core types was less successful when modeling referential activity in Italian. (See Mariani, Maskit, Bucci and De Coro, 2013). This led to a model that includes a much larger number of types (9596). The IWRAD covers approximately $97 \%$ of the tokens used in the sample from which the dictionary was derived and correlates between $r=.24$ and $r=.91$ with samples used to develop and test the model.

\subsection{The need for understanding common predictors of RA across languages.}

Successful computational models have been built for referential activity (RA) in several languages including: English (Bucci and Maskit, 2006); Italian (Mariani et al., 2013); Spanish (Roussos and O'Connel, 2005) and German (Ehrman, 1997); however, each model was built separately and without a common basis. The current study seeks to identify a model that may be applied across languages based on a common 
definition of the features associated with referential activity.

We address this question by identifying those predictors in English and Italian that are most strongly associated with RA scores and have shared meaning. We hope that this model will be validated in other languages and develop into a generalized model that may be applied across languages. The value of such a model is twofold. First, there is pragmatic value as we receive requests from psychotherapy researchers who are working in various languages for which no computational model exists. ${ }^{1}$ Second, the development of a generalized model provides a unique opportunity to study the construct validity of the referential process. To the degree that we can describe how the process of putting feelings into words plays out across languages we will have a more accurate description of this basic psychological process.

\section{Methods and Results}

\subsection{Spoken corpora.}

This study utilized segments from the same corpora that were used to build the English ( $\mathrm{N}=763$ segments) and Italian ( $\mathrm{N}=936$ segments) Weighted Referential Activity Dictionaries. The segments in the English sample had an average length of 163 words (SD 115) and in the Italian sample had an average length of 83 words (SD 60). Both corpora included psychotherapy and other spoken narrative material such as interviews, monologues, early memories and stories told to pictures. These materials were reliably segmented and scored for referential activity by judges following instructions from Bucci et al. (2004) and in Italian following a translation of this manual by De Coro and Caviglia (2000). All of the texts were scored for the four RA scales by at least two trained raters who had achieved high inter-rater reliability. The average of the scales was taken as the RA score. Detailed descriptions of the composition of these samples and scoring procedures may be found in Bucci \&

\footnotetext{
${ }^{1}$ We have received requests for measures of referential activity in: Bulgarian, French, German, Hebrew, Mandarin, Norwegian, Polish and Portuguese.
}

Maskit (2006), for the English sample and Mariani et al. (2013), for the Italian sample.

For the current study both the English and Italian corpora were subdivided into training, testing and validation subsets. The training subset (English $\mathrm{N}=362$, Italian $\mathrm{N}=472$ ) was used to develop the model in this study and the test subset (English $\mathrm{N}=209$, Italian $\mathrm{N}=272$ ) was used to test interim models. The validation subset (English $\mathrm{N}=192$, Italian $\mathrm{N}=192$ ) was reserved for final validation of the model.

\subsection{The Discourse Attributes Analysis Programs (DAAP) and (IDAAP).}

The Discourse Attributes Analysis Program (DAAP) is a computer-based text analysis system designed by Bernard Maskit, whose features include the use of both weighted and unweighted dictionaries. The Italian version of the software differs mainly in its ability to handle accented letters from a variety of text formats. The English version of the software is publicly available at: https://github.com/DAAP/DAAP09.6

\subsection{Existing referential process dictionaries.}

The phases of the referential process are operationalized by three main measures: disfluency (Arousal Phase), referential activity (Symbolizing Phase), and reflection (Reorganizing Phase). In addition there are several other dictionary based measures that are routinely used in studies of the referential process. These dictionaries were created using standard procedures for computerized text analysis that involve compiling word lists for a large source of texts and selecting items based on agreement among judges following the conceptual definitions of the dictionaries which follow below. The dictionaries listed below were created independently in English and Italian except where otherwise indicated.

Disfluency (DF): A limited set of six items that people use when struggling to communicate such as 'um', 'uh', 'hmmm', etc. Disfluent language is also associated with cognitive load and effort in speech planning (Bortfeld et al., 2001). In addition to matching types in the disfluency dictionary DAAP also counts incomplete 
words (indicated by transcribers using a dash), repeated words and repeated two word phrases as disfluencies.

Reflection (REF): A dictionary of 1436 words that concern how people think and communicate thoughts. This dictionary includes words referring to cognitive or logical functions, e.g., 'assume', 'think', 'plan'.

Affect (AFF): Words that concern how people feel and communicate feelings directly such as, 'angry', 'sad', and 'happy'. The global measure of these words is the 'Affect Sum' dictionary (AFFS 2452 words). These are further classified as 'Positive' (AFFP; 620 words), 'Negative' (AFFN; 1470 words) and 'Mixed' affect (AFFZ; 362 words) words. The definitions of positive and negative affect are self explanatory; mixed affect words are words that seem to have an affective or emotional loading, but are neither positive, nor negative, e.g., 'anticipate', 'overwhelmed', 'serious'.

Sensory Somatic (SENS): A set of 1936 words pertaining to bodily and or sensory experience, e.g., 'dizzy', 'eye', 'face', 'listen'.

Negation (NEG): A limited set of 26 items that people use when negating in communication. e.g., 'no', 'not', 'never'; this may be seen as a function of the logic mode. This dictionary was not created independently in Italian.

\subsection{Test of automated translation of existing dictionaries.}

In order to test how well dictionaries might work in direct translation by automated means, all of the dictionaries described above in English and Italian were translated using Google Translate (through Google Sheets). The translated dictionaries were then run on the corpora described above using the English and Italian versions of the DAAP. Tables 1 and 2 show the correlations of the native language to the translated dictionaries.

The translation of the dictionaries used here,

\footnotetext{
${ }^{2}$ Since there are core differences in typical words used as disfluencies in English, such as, 'like', and Italian, such as 'boh' the full dictionary in each language was compared to a common subset of lexical items, e.g., 'uh' and 'um'.
}

\begin{tabular}{lr}
\hline Dictionary & Correlation \\
\hline Negative Affect & $.75^{* * *}$ \\
Positive Affect & $.33^{* * *}$ \\
Mixed Affect & $.69^{* * *}$ \\
Affect Sum & $.66^{* * *}$ \\
Reflection & $.44^{* * *}$ \\
Sensory Somatic & $.62^{* * *}$ \\
Disfluency & $.66^{* * *}$ \\
WRAD \& IWRAD-T & $-.10^{* *}$ \\
\hline Note: $\mathrm{N}=763 ;{ }^{*} \mathrm{p}<.05 ;{ }^{* *} \mathrm{p}<.01 ;^{* * *} \mathrm{p}<.001$
\end{tabular}

Table 1: Pearson's correlations of English DAAP dictionaries with translated Italian DAAP dictionaries (translated using Google Translate).

\begin{tabular}{lr}
\hline Dictionary & Correlation \\
\hline Negative Affect & $.27^{* * *}$ \\
Positive Affect & $.57^{* * *}$ \\
Mixed Affect & $.34^{* * *}$ \\
Affect Sum & $.40^{* * *}$ \\
Reflection & $.33^{* * *}$ \\
Sensory Somatic & $.40^{* * *}$ \\
Disfluency & $.23^{* *}$ \\
IWRAD \& WRAD-T & $.16^{* * *}$ \\
\hline Note: $\mathrm{N}=936{ }^{*} \mathrm{p}<.05 ;{ }^{* *} \mathrm{p}<.01 ;{ }^{* * *} \mathrm{p}<.001$
\end{tabular}

Table 2: Pearson's correlations of Italian DAAP dictionaries with translated English DAAP dictionaries (translated using Google Translate).

based largely on content words including nouns, verbs and adjectives, showed moderate to strong correlations both from and to Italian. Though manual translation would likely show stronger results, these results indicate that even automated translation of these dictionaries shows good correspondence with the dictionaries created by native speakers. The two computational models of RA, which are more dominated by function words, though strongly correlated with Judges' RA in both languages (English $r(761)=$ $.53, p<.001 ;$ Italian $r(934)=.73, p<.001)$ were only weakly correlated and in the opposite of the expected direction in Italian (as shown in Tables 1 and 2). The translated dictionaries were also weakly related to judge scored RA (English $r(761)=-.03, p=.363$; Italian $r(934)=.04, p=.130)$. The difficulty of trans- 
lating these dictionaries based on style rather than content underscore the need for the current study.

\subsection{New model development.}

In order to develop a more universal model of referential activity, impact scores were created for the current English and Italian WRAD dictionaries by multiplying the WRAD weight for each dictionary term by the frequency with which it occurred in the corpus from which the dictionary was derived. These lists were then sorted by this impact score and compared. Words in the top 50 of both lists that had shared translated meaning were selected. Finally, the word 'uno' (ranked 97) was added as the masculine of 'una' (ranked 13), the word 'mia' (ranked 86) was added as the feminine of 'mio' (ranked 44) and the word 'ed' (ranked 99) was added as an additional translation of the English 'and'. Table 3 below shows the selected words along with the proportion of the corpus each represents. ${ }^{3}$

\subsection{New 'Universal Weighted Referential Activity Dictionary' (UWRAD) regression model.}

All types ${ }^{4}$ in Table 3 were entered into a regression model predicting the RA score for the Italian training set along with the following dictionaries translated to Italian from English using Google Translate: SENS, DF, REF, NEG, and AFFS. All entries that were significant at the .10 level or better were retained, all others were dropped. This left 'and', 'to', 'he / she', 'the', 'was', SENS and NEG in the final model shown in Table 4.

\subsection{New model performance.}

Table 5 shows the correlations of the UWRAD model with judge scored referential activity in each of the sub-samples of the two corpora.

\footnotetext{
${ }^{3}$ The number 50 was an arbitrary choice intended to include a high proportion of the positive predictors of RA in both languages.

${ }^{4}$ She / He was added as a single type all others were kept separate.
}

\begin{tabular}{|c|c|c|c|}
\hline English & Proportion & Italian & Proportion \\
\hline \multirow[t]{3}{*}{ and } & $4.3 \%$ & $\mathrm{e}$ & $2.7 \%$ \\
\hline & & ed & $0.1 \%$ \\
\hline & & poi & $0.6 \%$ \\
\hline \multirow[t]{6}{*}{ the } & $3.2 \%$ & il & $1.1 \%$ \\
\hline & & le & $0.6 \%$ \\
\hline & & gli & $0.4 \%$ \\
\hline & & lo & $0.5 \%$ \\
\hline & & $\mathrm{i}$ & $0.5 \%$ \\
\hline & & la & $1.6 \%$ \\
\hline \multirow[t]{3}{*}{$\mathrm{a}$} & $1.9 \%$ & un & $1.8 \%$ \\
\hline & & una & $1.2 \%$ \\
\hline & & uno & $0.2 \%$ \\
\hline \multirow[t]{2}{*}{ my } & $0.8 \%$ & mio & $0.5 \%$ \\
\hline & & mia & $0.6 \%$ \\
\hline all & $0.3 \%$ & tutti & $0.2 \%$ \\
\hline from & $0.2 \%$ & da & $0.6 \%$ \\
\hline \multirow[t]{3}{*}{ to } & $2.9 \%$ & $\mathrm{a}$ & $1.8 \%$ \\
\hline & & al & $0.3 \%$ \\
\hline & & per & $1 \%$ \\
\hline in & $1.3 \%$ & in & $1.2 \%$ \\
\hline she & $2.1 \%$ & lei & $0.4 \%$ \\
\hline he & $1.2 \%$ & lui & $0.5 \%$ \\
\hline they & $0.7 \%$ & sono & $1 \%$ \\
\hline \multirow[t]{2}{*}{ me } & $0.6 \%$ & mi & $1.6 \%$ \\
\hline & & me & $0.5 \%$ \\
\hline \multirow[t]{2}{*}{ there } & $0.5 \%$ & là & $1.6 \%$ \\
\hline & & lì & $0.2 \%$ \\
\hline \multirow[t]{2}{*}{ was } & $1.6 \%$ & era & $0.6 \%$ \\
\hline & & ero & $0.2 \%$ \\
\hline \multirow[t]{3}{*}{ had } & $0.6 \%$ & aveva & $0.2 \%$ \\
\hline & & avevo & $0.2 \%$ \\
\hline & $22 \%$ & & $24 \%$ \\
\hline
\end{tabular}

Table 3: Predictors of RA with shared translated meaning. These types were selected by comparing the top 50 positive predictors of referential activity in English and Italian from previous computational models.

As for the UWRAD's relation to other indices of the referential process, the model correlated $r(761)=-.38, p<.001$ with Reflection in English and $r(934)=-.28, p<.001$ in Italian. UWRAD also correlated $r(761)=$ $-.16, p<.001$ with Disfluency ${ }^{5}$ in English and

\footnotetext{
${ }^{5}$ Disfluency was modified for this comparison as discussed above by removing all language specific indicators.
} 


\begin{tabular}{|c|c|}
\hline & Dependent variable: \\
\hline & RA Score \\
\hline and & $\begin{array}{c}8.370^{* * *} \\
(2.240)\end{array}$ \\
\hline to & $\begin{array}{l}6.780^{* *} \\
(2.580)\end{array}$ \\
\hline he / she & $\begin{array}{c}6.880 \dagger \\
(3.910)\end{array}$ \\
\hline the & $\begin{array}{c}8.470^{* * *} \\
(2.060)\end{array}$ \\
\hline was & $\begin{array}{c}16.300^{* *} \\
(6.120)\end{array}$ \\
\hline Sensory Somatic & $\begin{array}{l}6.120^{*} \\
(2.430)\end{array}$ \\
\hline Negation & $\begin{array}{c}-12.900^{* * *} \\
(2.140)\end{array}$ \\
\hline Constant & $\begin{array}{c}4.140^{* * *} \\
(0.180)\end{array}$ \\
\hline Observations & 472 \\
\hline $\mathrm{R}^{2}$ & 0.197 \\
\hline Adjusted $\mathrm{R}^{2}$ & 0.185 \\
\hline Residual Std. Error & $1.320(\mathrm{df}=464)$ \\
\hline F Statistic & $16.200^{* * *}(\mathrm{df}=7 ; 464)$ \\
\hline
\end{tabular}

Note: $\quad \dagger \mathrm{p}<.10 ;{ }^{*} \mathrm{p}<.05 ;{ }^{* *} \mathrm{p}<.01 ;{ }^{* * *} \mathrm{p}<.001$

Table 4: 'Universal Weighted Referential Activity' (UWRAD) model.

$r(934)=-.23, p<.001$ in Italian. This pattern of correlations, which is typically observed in studies of the referential process, indicate that the Arousal, Symbolizing and Reorganizing phases are distinct from one another ${ }^{6}$ and thus provide support for the construct validity

\footnotetext{
${ }^{6}$ Correlations of Reflection and Disfluency were $r(761)=-.02, p=.567$ and $r(934)=.02, p=.571$, suggesting that these dimensions are orthogonal.
}

\begin{tabular}{lr}
\hline Sample & Correlation \\
\hline English $(\mathbf{N}=\mathbf{7 3 6})$ & $.43^{* * *}$ \\
Training $(\mathrm{n}=362)$ & $.41^{* * *}$ \\
Testing $(\mathrm{n}=209)$ & $.44^{* * *}$ \\
Validation $(\mathrm{n}=192)$ & $.45^{* * *}$ \\
Italian $(\mathbf{N}=\mathbf{9 3 6})$ & $.41^{* * *}$ \\
Training $(\mathrm{n}=472)$ & $.44^{* * *}$ \\
Testing $(\mathrm{n}=272)$ & $.38^{* * *}$ \\
Validation $(\mathrm{n}=192)$ & $.36^{* * *}$ \\
\hline Note: ${ }^{*} \mathrm{p}<.05 ;{ }^{* *} \mathrm{p}<.01 ;{ }^{* * *} \mathrm{p}<.001$
\end{tabular}

Table 5: Pearson's correlations of the 'Universal Weighted Referential Activity Dictionary' (UWRAD) with judge scored RA for all subsets in English and Italian

of the UWRAD as developed here.

\section{Discussion}

This study identified a single model comprised of seven common components that accounted for $13 \%$ to $20 \%$ of the variance in judges' scores of referential activity in two languages. As this model was able to function in translation between English and Italian, which have quite different lexical structures, it holds promise to translate into other languages as well. Future research will explore this modeling strategy in additional languages by comparing the model identified here to the scoring of referential activity by native speakers in similar corpora to those utilized here. Future research will also test this model for stability in additional samples of English and Italian.

While this study shows promise to facilitate the development of computational models of referential activity in other languages it also represents an opportunity to better understand the construct of referential activity. We believe that the components identified by this model support the idea that referential activity represents the language of scene construction. As evidence, the model makes use of definite object references ('the') sensate and experience near content (Sensory Somatic), the movement of actors and objects ('to') and orientation in time ('was'), all of which are consistent with the detailed description of images and events. A higher density of 
'and' suggests that such texts may be similarly dense in details and events which require connection to one another in the context of a scene.

The inclusion of the third person singular animate pronouns 'he' and 'she' is consistent with an emphasis on concrete immediate experience with other people as opposed to internal reflection and consideration which may be more likely to involve a more reflective or abstract point of view. Similarly, negations as strong negative predictors of RA suggest that when speakers are using high RA language they are not engaged in reconsideration and qualification involving logical operations.

Scene construction is consistent with the idea of the Symbolizing Phase of the referential process which suggests that our felt experience is most effectively conveyed by telling about the events and images that give rise to a feeling so that others may become engaged in the event in their own bodily, sensory and emotional experience. This basic function is central to communicating emotional experience to others, and to making sense of our own experience. Such a dimension is necessarily important for understanding emotion in interpersonal communication in clinical contexts such as psychotherapy and the diagnosis of problems with memory and emotion, and in non-clinical contexts such as inspiration in political speech, clarity in effective teaching, or connection in interpersonal relationships.

\section{References}

Donna Rose Addis, Alana T Wong, and Daniel L Schacter. 2008. Age-related changes in the episodic simulation of future events. Psychological science, 19(1):33-41, January.

Eva Appelman. 2000. Attachment experiences transformed into language. American Journal of Orthopsychiatry, 70(2):192-202.

H Bortfeld, S. D. Leon, J. E. Bloom, M. F. Schober, and S. E. Brennan. 2001. Disfluency rates in conversation: Effects of age, relationship, topic, role, and gender. Language and Speech, 44(2):123-147, June.

Wilma Bucci and Bernard Maskit. 2006. A weighted dictionary for referential activity. In James G.
Shanahan, Yan Qu, and Janyce Wiebe, editors, Computing attitude and affect in text: Theory and applications, volume 20 of The Information Retrieval Series. Springer-Verlag, Berlin/Heidelberg.

Wilma Bucci and Bernard Maskit. 2007. Beneath the surface of the therapeutic interaction: the psychoanalytic method in modern dress. Journal of the American Psychoanalytic Association, 55(4):1355-1397, December.

Wilma Bucci, R. Kabasakalian-McKay, and The RA Research Groups. 2004. Scoring Referential Activity instructions for use with transcripts of spoken texts.

Wilma Bucci, Bernard Maskit, and Leon Hoffman. 2012. Objective measures of subjective experience: the use of therapist notes in process-outcome research. Psychodynamic psychiatry, 40(2):30340, June.

Wilma Bucci. 1984. Linking words and things: Basic processes and individual variation. Cognition, 17(2):137-153, July.

Wilma Bucci. 1997. Psychoanalysis and cognitive science: A multiple code theory. Guilford Press, New York, NY, US.

Herbert H. Clark. 1996. Using language. Cambridge University Press, Cambridge.

A. De Coro and G. Caviglia. 2000. La valutazione dell'Attivita' Referenziale.

Michael Dodd and Wilma Bucci. 1987. The relationship of cognition and affect in the orientation process. Cognition, 27(1):53-71, October.

Susanne Ehrman. 1997. Computer measures of the cycles model: The german version. Ph.D. thesis, Adelphi University.

Phoebe C Ellsworth and Eddie M W Tong. 2006. What does it mean to be angry at yourself? Categories, appraisals, and the problem of language. Emotion (Washington, D.C.), 6(4):572-86, November.

Suzanne Fleischman. 1990. Tense and narrativity: From medieval performance to modern fiction. Austin: University of Texas Press.

Polly Fretter, Wilma Bucci, Jessica Broitman, George Silberschatz, and John Curtis. 1994. How the Patient's Plan Relates to the Concept of Transference. Psychotherapy Research, 4(1):5872 , January.

Mardi J. Horowitz, Charles Stinson, Deborah Curtis, and Mary Ewert. 1993. Topics and signs: Defensive control of emotional expression. Journal of Consulting and Clinical Psychology, 61(3):421430 . 
Lisa Jepson and Wilma Bucci. 1999. Object relations and referential activity in physically abused adolescents. Adolescence, 34:780-792.

Robert J. Langs, Wilma Bucci, Andrea L. Udoff, Gail Cramer, and Lenore Thomson. 1993. Two methods of assessing unconscious communication in psychotherapy. Psychoanalytic Psychology, 10(1):1-16.

Katie Lewis, Sean Murphy, and Yuko Hanakawa. 2009. Uncovering episodic memory through linguistic measures in schizophrenia. In Poster session at the Association for Psychological Science Annual Convention, San Francisco.

Rachele Mariani, Bernard Maskit, Wilma Bucci, and Alessandra De Coro. 2013. Linguistic measures of the referential process in psychodynamic treatment: the English and Italian versions. Psychotherapy research: Journal of the Society for Psychotherapy Research, 23(4):430-47, January.

Bernard Maskit, Wilma Bucci, and Sean Murphy. 2014. Computer based measures of referential activity and their use as measures of episodic memory.

Bernard Maskit, Wilma Bucci, and Sean Murphy. 2015. A computer program for tracking the evolution of a psychotherapy treatment. In Computational Linguistics and Clinical Psychology Workshop at NAACL HLT 2015.

Erhard Mergenthaler and Wilma Bucci. 1999. Linking verbal and non-verbal representations: Computer analysis of referential activity. British Journal of Medical Psychology, 72(3):339-354, September.

Kristin Nelson and Michael Polignano. 2009. Referential activity in negative episodic 'flashbulb' memories from patients. In Poster session at the Association for Psychological Science Annual Convention, San Francisco, CA.

Kristin L. Nelson, Damian J. Moskovitz, and Hans Steiner. 2008. Narration and Vividness as Measures of Event-Specificity in Autobiographical Memory. Discourse Processes, 45(2):195-209, March.

Mark B Powers, Jacqueline M Halpern, Michael P Ferenschak, Seth J Gillihan, and Edna B Foa. 2010. A meta-analytic review of prolonged exposure for posttraumatic stress disorder. Clinical psychology review, 30(6):635-41, August.

Andres Roussos and Manuela O'Connel. 2005. Construcción de un diccionario ponderado en español para medir la Actividad Referencial. Revista del Instituto de Investgaciones de la Facultad de Psicología / UBA, 10.(2):99-119.
Nicolas Samstag. 1997. A meta-analysis of referential activity. Dissertation Abstracts International: Section B: The Sciences and Engineering, 57(9-B), 5 .

Endel Tulving. 1972. Episodic and semantic memory. In Organization of memory, volume 1, pages 381-403.

J C Watson. 1996. The relationship between vivid description, emotional arousal, and in-session resolution of problematic reactions. Journal of consulting and clinical psychology, 64:459-464. 